\title{
Study on a Compression Algorithm of SAR Data after Range Focusing
}

\author{
Shang-Chun Zeng ${ }^{1,}$, Yun-Xia Xie ${ }^{2}$, Yi-Xian Chen ${ }^{1}$, Xian-Lin Deng ${ }^{3}$ and Wang Huazhang ${ }^{1}$ \\ ${ }^{1}$ College of Electrical \& Information Engineering, Southwest University for Nationalities, Chengdu, 610041, China \\ ${ }^{2}$ School of Science, Sichuan University of Science \& Engineering, Zigong, 643000, China \\ ${ }^{3}$ Department of Electronic Information, Chongqing College of Electronic Engineering, Chongqing, 401331, China
}

\begin{abstract}
It is difficult to directly compress the raw data of synthetic aperture radar because of its low relativity. In this paper, a new algorithm is put forward. Firstly, range focusing is imposed to SAR raw data, which makes it have comparatively high relativity, secondly, a linear prediction is performed along the azimuth, and lastly, block adaptive quantization is performed on the prediction difference series. By using a set of real SAR raw data, compression and decompression are performed respectively. The SQNR and SDNR are achieved. The images corresponding to the three algorithms are gained. The experiments manifest that with same bit rate, SQNR and SDNR of the algorithm proposed in this paper surpass that of BAQ algorithm. The calculation in this paper is far less than that of compression method after range focusing advised in corresponding reference. The algorithm proposed in this paper has a certain practical value.
\end{abstract}

Keywords: Range focusing, linear prediction, signal to noise ratio, bit rate, block adaptive quantization.

\section{INTRODUCTION}

Synthetic aperture radar (SAR) is a new type of radar system, it has the two-dimensional (range and azimuth) high resolution features, as well as broad and all-weather imaging characteristics, which are unmatched by conventional radar. Modern airborne synthetic aperture radar system has a hardware processing system which includes a transmitting unit, a receiving unit and an analog to digital conversion, etc., as well as real time data transmission or storage devices. A major problem of SAR system is the high data rates which are difficult to real-time transmit, so it is necessary to reduce SAR raw data rates to meet the limited downlink bandwidth. Due to the high entropy characteristics of SAR raw data, lossless compression algorithm is difficult to meet the large compression ratio requirements, so loss compression techniques are commonly used at present.

A variety of compression algorithms has been studied currently in the area of SAR raw data compression, such as BAQ [1], UPQ [2], vector quantization [3], FFT-BAQ [4] and TCQ [5], etc.. The correlation is very low for SAR raw data, so directly compressing SAR raw data is more difficult. Not focusing complex range, it can make the SAR data have a strong correlation in azimuth, which makes SAR raw data become easy to compress. This is an attractive research direction of SAR raw data compression field. Literature [6]

*Address correspondence to this author at the College of Electrical \& Information Engineering, Southwest University for Nationalities, Chengdu, 610041, China; Tel: (+86)13693412034; E-mail: zsc5100@sina.com studies variable rate vector quantization after range focusing for SAR raw data compression, the performance of this method is better than the traditional unfocused SAR raw data compression algorithms. As a result of vector quantization, the computational complexity of this method is relatively large. Literature [7] uses TCVQ coding to compress the SAR data after range focusing, in order to reduce the computational complexity, the shorter code-words than those in literature [6] are used, the reduction of the codeword length will inevitably lead to a decrease in performance, but TCVQ algorithm has a more rational codebook space division, which reduces the quantization error. Therefore, the performance of the method used in literature [7] is comparative to that of the method used in literature [6], but has slightly smaller computational complexity. The computation of vector quantization increases exponentially with the codeword length, which greatly limits the use of these two methods in the real-time transmission of SAR raw data. Therefore, as to the SAR data after range focusing, it is necessary to study algorithms with smaller complexity. In this paper, range focusing is imposed to SAR raw data, then a linear prediction is performed along the azimuth, and finally block adaptive quantization is used to the prediction difference series. The experiments manifest that the calculation in this paper is far less than that of compression method after range focusing advised in literature [6], but the performance is slightly lower.

\section{ALGORITHM ANALYSIS}

SAR echo data is two-dimensional complex matrix along range and azimuth, in addition, real part and imaginary part 
Table 1. Line complexity.

\begin{tabular}{|c|c|c|c|c|c|}
\hline Order & $\mathbf{1}$ & $\mathbf{2}$ & $\mathbf{3}$ & $\mathbf{4}$ & $\mathbf{5}$ \\
\hline \hline SAR raw data & 1.257 & 1.395 & 1.418 & 1.411 & 1.423 \\
\hline Data after range focusing & 1.045 & 1.213 & 1.403 & 1.413 & 1.413 \\
\hline
\end{tabular}

Table 2. Column complexity.

\begin{tabular}{|c|c|c|c|c|c|}
\hline Order & $\mathbf{1}$ & $\mathbf{2}$ & $\mathbf{3}$ & $\mathbf{4}$ \\
\hline \hline SAR raw data & 1.307 & 1.507 & 1.328 & 1.449 & 1.441 \\
\hline Data after range focusing & 1.466 & 1.515 & 1.499 & 1.439 & 1.379 \\
\hline
\end{tabular}

are independent of each other, both of which are zero-mean Gaussian distribution with the same variance. Therefore, it is possible to process real part and imaginary part respectively, the size of data block needing to deal with is $2048 \times 2048$ (azimuth $\times$ range). SAR focusing process is usually carried out in two steps: (1) range focusing; (2) azimuth focusing which depends on range. Azimuth focusing is more complicated, and the reference function of which is related to slant range $r$, while the reference function (related to transmission signal) of range focusing corresponding to each echo is the same, which is equivalent to the echo signal passing from an identical linear filter. Thus only range focusing is performed in this paper. Line complexity and column complexity are adopted to evaluate the correlation of SAR raw data. For a SAR raw data block (size $N \times N), L(k)$ is used to represent line complexity (namely azimuth complexity), while $C(k)$ is used to represent column complexity (namely range complexity). The formulas of $L(k)$ and $C(k)$ are as follows:

$$
\begin{aligned}
& L(k)=\left[\frac{1}{(N-k) \times N} \sum_{i=0}^{N-k-1} \sum_{j=0}^{N-1}(A(i, j)-A(i+k, j))^{2}\right]^{1 / 2} \\
& C(k)=\left[\frac{1}{N \times(N-k)} \sum_{i=0}^{N-1} \sum_{j=0}^{N-k-1}(A(i, j)-A(i, j+k))^{2}\right]^{1 / 2}
\end{aligned}
$$

In the above two formulas, $A(i, j)$ represents the value of SAR raw data at point $(i, j)$ (real part or imaginary part), $k$ is the order.

Expand (1), we get:

$$
L(k)=\left[\frac{1}{(N-k) \times N} \sum_{i=0}^{N-k-1} \sum_{j=0}^{N-1}\left(\begin{array}{l}
A^{2}(i, j)+A^{2}(i+k, j)- \\
2 A(i, j) A(i+k, j)
\end{array}\right)\right]^{1 / 2}
$$

In the above formula, the cross term $2 A(i, j) A(i+k, j)$ represents line correlation. It can be seen, for the same data block, the smaller $L(k)$, the stronger the line correlation, and $C(k)$ is similar.
Table 1 and Table 2 show $L(k)$ and $C(k)$ for a block of normalized SAR raw data and range focused SAR data respectively. Because the correlation difference between real part and imaginary part for the same SAR data block is very small, the two tables give only the correlation value of real part. As can be seen, after performing range focusing on a SAR raw data block, the line complexity changes relatively large, but little change with the column complexity. In other words, the SAR data after range focusing show a strong correlation along azimuth, while the correlation along range is still very weak.

SAR data after range focusing show a relatively strong correlation along azimuth, it already has some characteristics of image, referred to as quasi-image. Fig. (1) is an amplitude image of SAR data after range focusing. As can be seen, the amplitude image of SAR data after range focusing has some bright lines along azimuth, which shows that energy goes through convergence and a strong correlation appears along azimuth.

SAR data after range focusing have a greater dynamic range than raw data, so it is relatively difficult to compress them. Learn from the thinking of the BAQ algorithm, still large data block (2048X2048) is divided into small data blocks (32X32), then normalization is performed on each of small data blocks (32X32). Distance focusing is equivalent to a linear transformation, so it does not change the Gaussian distribution of SAR raw data.

Theoretically, with the increase in the number of prediction order, the calculation also increases. As can be seen from Table 1, line complexity changes little apart two points (i.e. 3rd order). So a 3rd order linear prediction is used in this paper along azimuth. When the different predictors are designed for different range gates, the computation can become too large, which is not conducive for real-time processing. In this paper, a simplified approach is adopted: a predictor is designed every 512 range gates. Experiments show that the error by such processing is very small. Furthermore, the SAR data after range focusing have a large dynamic range, therefore, block normalization (block size $32 \times 32$ ) should be made firstly then linear prediction is to be performed along azimuth. Because of very wide SAR imaging areas, backscatter coefficients of different targets are not same, therefore, some of the data blocks will deviate from 


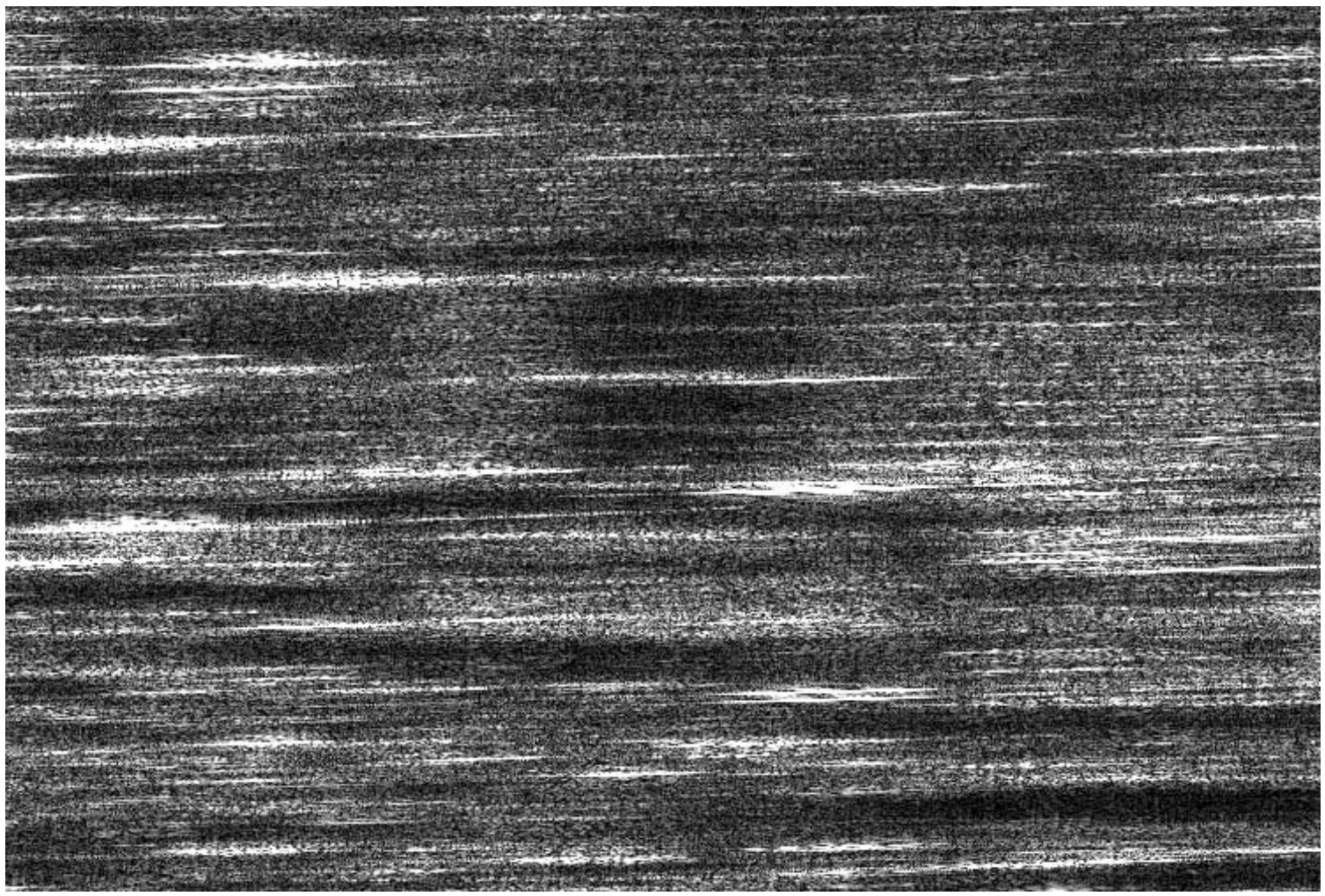

Fig. (1). Amplitude image of SAR data after range focusing.

Table 3. Performance parameter of the three compression method in different bit rate.

\begin{tabular}{|c|c|c|c|c|c|c|c|c|c|c|}
\hline \multirow{2}{*}{ Algorithm } & \multirow{2}{*}{$\begin{array}{c}\text { Compression } \\
\text { Ratio }\end{array}$} & \multirow{2}{*}{$\begin{array}{c}\text { Bit Rate } \\
\text { Bits/Sample }\end{array}$} & \multirow{2}{*}{$\frac{\text { SQNR }}{\text { dB }}$} & \multirow{2}{*}{$\begin{array}{c}\text { SDNR } \\
\mathrm{dB}\end{array}$} & \multicolumn{2}{|c|}{ Resolution/m } & \multicolumn{2}{|c|}{ PSLR/dB } & \multicolumn{2}{|c|}{$\operatorname{ISLR}(d B)$} \\
\hline & & & & & Range & Azimuth & Range & azimuth & Range & azimuth \\
\hline BAQ & 8 & 1 & 6.56 & 8.08 & 3.53 & 2.62 & -8.77 & -10.91 & -5.67 & -6.24 \\
\hline BAQ & 4 & 2 & 11.48 & 14.28 & 3.37 & 2.57 & -9.1 & -11.09 & -6.15 & -7.01 \\
\hline BAQ & 2.67 & 3 & 16.84 & 20.13 & 3.31 & 2.59 & -9.52 & -10.91 & -6.61 & -6.93 \\
\hline literature [6] & 8 & 1 & 8.57 & 9.87 & 3.6 & 2.53 & -8.64 & -11.55 & -6.41 & -6.03 \\
\hline literature [6] & 4 & 2 & 14.25 & 16.68 & 3.38 & 2.62 & -9.3 & -10.94 & -6.53 & -6.84 \\
\hline literature [6] & 2.67 & 3 & 20.11 & 23.04 & 3.32 & 2.61 & -9.51 & -10.68 & -6.35 & -6.95 \\
\hline this paper & 8 & 1 & 8.22 & 9.56 & 3.58 & 2.54 & -8.72 & -11.44 & -6.32 & -6.01 \\
\hline this paper & 4 & 2 & 13.71 & 16.12 & 3.36 & 2.61 & -9.26 & -10.97 & -6.55 & -6.71 \\
\hline this paper & 2.67 & 3 & 19.34 & 22.24 & 3.35 & 2.63 & -9.56 & -10.74 & -6.84 & -6.31 \\
\hline SAR raw data & & & & & 3.32 & 2.61 & -9.51 & -10.64 & -6.74 & -6.92 \\
\hline
\end{tabular}

Gaussian distribution. Literature [8] points out that if the original series is progressive Gaussian distribution, the difference series obtained from prediction has Gaussian distribution characteristics more than the original series. Moreover, the number of values which are close to zero in the difference series significantly increases, and the dynamic range becomes smaller, which is more beneficial to quantify. The block adaptive quantization is performed on the difference series obtained from linear prediction, the block size is $32 \times 32$, each block of data uses identical quantization bit.
Therefore, linear prediction-block adaptive quantization is adopted in this paper.

\section{SIMULATION RESULTS}

In this paper, a set of measured side-looking airborne SAR raw data is used (strip mode), and the compression algorithm proposed in this paper is performed. Since BAQ algorithm is more mature than other practical SAR raw data compression algorithms, the results of the algorithm pro- 


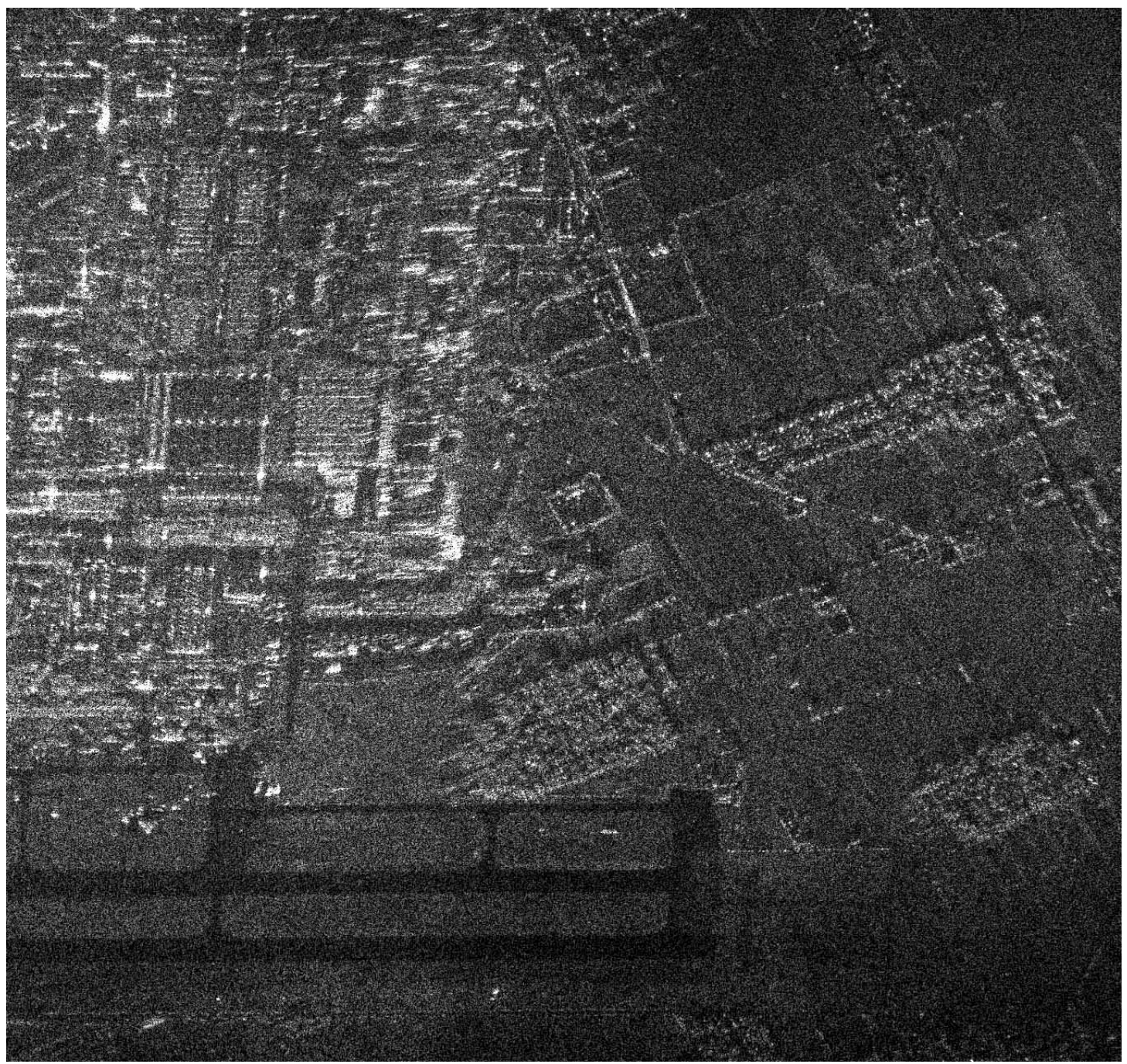

Fig. (2). Image of the BAQ Algorithm (bit rate 2 bits/sample).

posed in this paper are compared with BAQ algorithm. In addition, the two algorithms proposed in literature [6] and literature [7] respectively give equivalent performance, for further comparison, the performance parameters of algorithm proposed in literature [6] are calculated. Table 3 shows the calculation results of the three algorithms at different bit rates.

In this paper, data domain signal-to-noise ratio (SQNR) and the image domain signal-to-noise ratio (SDNR) are regarded as compression evaluation parameters. At the same time, the spatial resolution ( $\rho$ ), PSLR (Peak Side lobe Ratio) and ISLR (Integral Side lobe Ratio) of image is calculated [9]. It is clearly seen, in the same bit rate, SQNR and SDNR of the algorithm mentioned in this paper surpass that of BAQ algorithm, but slightly lower than the algorithm mentioned in literature [6]. At the same time, it can be seen the little change in resolution, PSLR and ISLR in the different bit rate. Which shows that data compression almost does not affect the resolution of SAR image, which also shows that SAR image is not sensitive to the number of quantization bits of SAR raw data.
Fig. (2) shows the image obtained from BAQ algorithm. Fig. (3) shows the image obtained from the algorithm proposed in literature [6]. Fig. (4) shows the image obtained from the algorithm proposed in this paper. Due to space limitations, the images are given only when bit rate is 2 bits/sample.

\section{ALGORITHM COMPLEXITY ANALYSIS}

Set $N_{r}$ is the data length along range, $N_{a}$ is the data length along azimuth, $N$ is the data block length of original data in the normalization operation, and $p$ is the order of linear predictor. For the computational complexity of algorithm, addition, comparison and multiplication can be completed in the same clock cycle by DSP. Therefore, each addition, comparison and multiplication of floating-point in the algorithm can be seen as a single operation. For BAQ algorithm, to quantize a sampled value, it needs 2 operations to estimate mean value 2 operations to normalize data, and $2^{b}$ ( $b$ represents the number of quantization bits) operations to execute Lloyd-Max coding. Range focusing in this paper is 


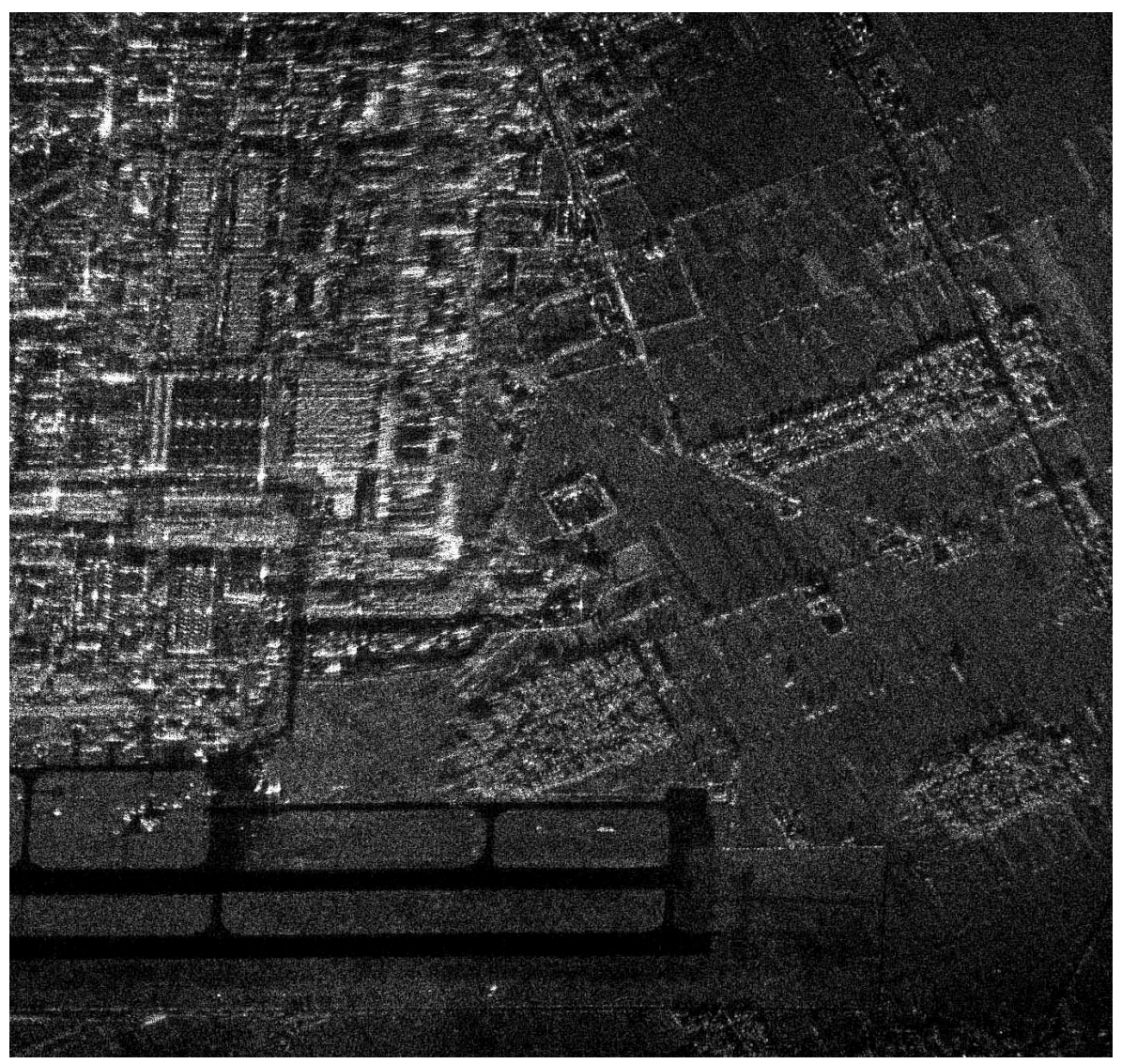

Fig. (3). Image of the literature [6] algorithm (bit rate 2 bits/sample).

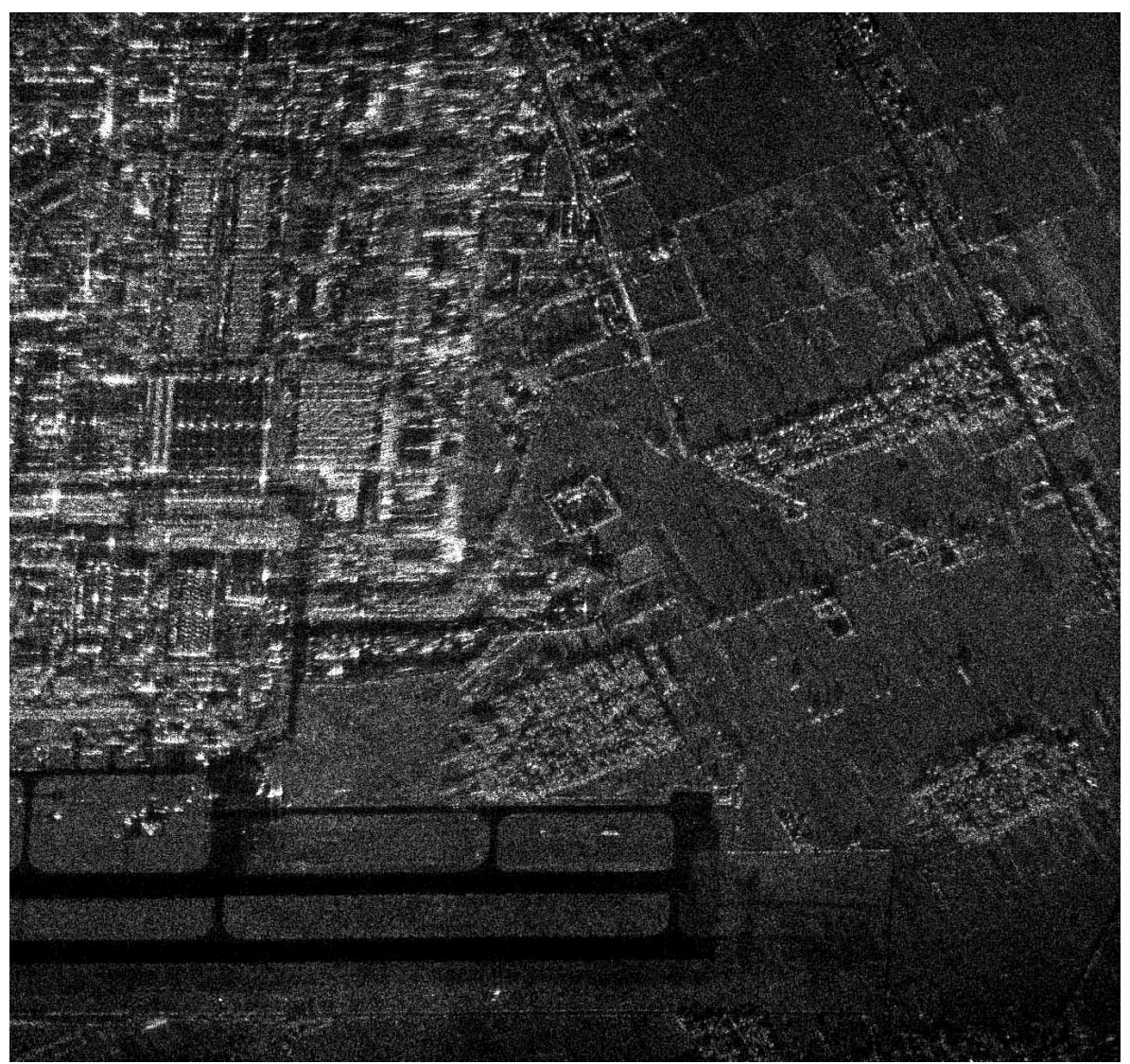

Fig. (4). Image of the algorithm proposed in this paper (bit rate 2 bits/sample). 
Table 4. Algorithm Complexity Analysis.

\begin{tabular}{|c|c|c|}
\hline \multirow{2}{*}{ Algorithm } & Storage Space (Floating Point) & Amount of Calculation \\
\cline { 2 - 3 } & RAM & (Operation/Sample) \\
\hline \hline BAQ & $2 \times N_{r} \times N$ & $2^{b}+4$ \\
\hline literature [6] & $2 \times N_{r} \times N$ & $12 \log _{2}^{N_{r}}+2 L \cdot 2^{R L}+3$ \\
\hline this paper & $2 \times N_{r} \times N_{a}$ & $12 \log _{2}^{N_{r}}+2 p+2^{b}+9$ \\
\hline
\end{tabular}

Table 5. The Storage Space, the Amount of Calculation and the Computation time of the Three Algorithms.

\begin{tabular}{|c|c|c|c|}
\hline \multirow{2}{*}{ Algorithm } & Storage Space (Floating Point) & Amount of Calculation & Computing Time \\
\cline { 2 - 4 } & RAM & (Operation/Sample) & (s) \\
\hline \hline BAQ & 131072 & 2183 & 0.23 \\
\hline literature [6] & 131072 & 151 & 5.36 \\
\hline this paper & 8388608 & 1.17 \\
\hline
\end{tabular}

equivalent to convolution, therefore, each echo data needs $N_{r}^{2}$ complex multiplications and $N_{r}^{2}$ complex additions, and each complex multiplication requires 6 operations, so the operations of each floating-point are as follows:

$\frac{N_{r}^{2} \times 6+N_{r}^{2} \times 2}{2 N_{r}}=4 N_{r}$

If using fast Fourier transform, the convolution of the time domain can be converted to the product of frequency domain, corresponding to each floating-point the operations are reduced to:

$$
\frac{\left(2 N_{r} \log _{2}^{N_{r}} \times 2+N_{r}\right) \times 6}{2 N_{r}}=\left(4 \log _{2}^{N_{r}}+1\right) \times 3
$$

For the $p$-th order linear predictor, $2(p+1)$ operations are needed for each floating-point.

For block adaptive quantization of prediction difference series, each floating-point number requires $\left(2^{b}+4\right)$ operations. Therefore, the number of operations required by the algorithm of this article for each floating-point is:

$\left(4 \log _{2}^{N_{r}}+1\right) \times 3+2(p+1)+4+2^{b}=12 \log _{2}^{N_{r}}+2 p+2^{b}+9$

Vector quantization is performed on the SAR data after range focusing as described in literature [6]. Set bit rate is $R$, the length of the codeword is $L$, then the operations of each component (floating point) in codeword searching process are $2 L \cdot 2^{R L}$. Therefore, the number of operations for each floating-point in the algorithm is:

$\left(4 \log _{2}^{N_{r}}+1\right) \times 3+2 L \cdot 2^{R L}=12 \log _{2}^{N_{r}}+2 L \cdot 2^{R L}+3$
On the space complexity of algorithms view, for BAQ algorithm, RAM storage area is mainly used to store raw data, estimate mean and variance of data block, and normalize data. Since a simplified method is used to estimate mean and variance, it spends less memory. In literature [6], because normalization process is applied to SAR data after range focusing, therefore, RAM storage area is the same as that of BAQ algorithm. For the algorithm proposed in this paper, RAM storage area is mainly used to store SAR data after range focusing and prediction difference series along azimuth. The size of cache space and computational complexity for three algorithms is shown in Table 4.

In this experiment, some variable values in Table $\mathbf{4}$ are as follows: $N_{r}=N_{a}=2048, N=32, b=R=2, L=4, p=3$. The storage space, the amount of computation and the computation time of microcomputer are given quantitatively in Table 5. It can clearly be seen, the storage space required by the algorithm proposed in this paper is larger than that of the algorithm proposed in literature [6], but the amount of computation and computing time are greatly reduced.

\section{CONCLUSION}

The SAR raw data after range focusing show a strong correlation in azimuth, which facilitates data compression. In this paper, firstly range focusing is imposed to SAR raw data, secondly a linear prediction is performed along the azimuth, and lastly block adaptive quantization is performed on the prediction difference series. The experiments manifest that with the same bit rate, SQNR and SDNR of the algorithm proposed in this paper surpass that of BAQ algorithm, but are found to be slightly lower than that of the algorithm proposed in literature [6]. When bit rate is 1, 2 and 3 bits/sample respectively, SQNR of the algorithm proposed in this paper 
is 1.66-2.50 dB higher than that of BAQ algorithm, and SDNR of the algorithm proposed in this paper is $1.48-2.11$ $\mathrm{dB}$ higher than that of BAQ algorithm. At the same time, it can be seen that SQNR of the algorithm proposed in this paper is $0.35-0.77 \mathrm{~dB}$ lower than that of the algorithm proposed in literature [6], and SDNR of the algorithm proposed in this paper is $0.31-0.80 \mathrm{~dB}$ lower than that of the algorithm proposed in literature [6]. Since scalar quantization is used to the prediction difference series, compared with literature [6], the computational complexity of the algorithm proposed in this paper greatly reduces, which is very beneficial to real-time requirements for SAR raw data compression. The algorithm proposed in this paper has a certain practical value.

\section{CONFLICT OF INTEREST}

The authors confirm that this article content has no conflicts of interest.

\section{ACKNOWLEDGEMENTS}

This project was supported by Sichuan University of Science \& Engineering (Grant No.2013RC08). Additionally, this work was supported by the Research Project of State Ethnic Affairs Commission with the number 12XNZ016.

\section{REFERENCES}

[1] R. Kwok, and W.T.K. Johnson, "Block adaptive quantization of Magellan SAR data", IEEE Transactions on Geoscience and Remote Sensing, vol. 27, no. 4, pp. 375-383, 1989.

[2] Y. M. Zhu, D. Y. Zhu, and Z. D. Zhu, "An improved algorithm in the application of SAR raw data compression", Airborne Radar, vol. 1, no. 145, pp. 25-30, 2004.

[3] Z. H. Guan, D. Y. Zhu, and Z. D. Zhu, "Compression of SAR raw data by block adaptive spherical vector quantization", Acta Aeronoutica et Astronautica Sinica, vol. 27, no. 1, pp. 82-86, 2006.

[4] U. Benz, K. Strodl, and A. Moreia, "A comparison of several algorithms for SAR raw data Compression", IEEE Transactions on Geoscience and Remote Sensing, vol. 33, no. 5, pp. 1266-1276, 1995.

[5] M.W. Marcellin, and T. R. Fischer, "Trellis coded quantization of memoryless and Gauss-Markov sources", IEEE Transactions on Communications, vol. 38, no. 1, pp. 82-93, 1990.

[6] G. Poggi, A. R. P. Ragozini, and L. Verdoliva, "Compression of SAR data through range focusing and variable-rate vector quantization", IEEE Transactions on Geoscience and Remote Sensing, vol. 38, no. 3, pp. 1282-1289, 2000.

[7] C. D'Elia, G. Poggi, and L. Verdoliva, "Compression of SAR data through range focusing and variable-rate trellis-coded quantization", IEEE Transactions on Image Processing, vol. 10, no. 9, pp. 1278-1286, 2001.

[8] E. Magli, and G. Olmo, "Lossy predictive coding of SAR raw data", IEEE Transactions on Geoscience and Remote Sensing, vol. 41, no. 5, pp. 977-987, 2003.

[9] X. J. Wang, H. Sun, and B. Guan, "Evaluation for coherent speckle suppression filters of SAR images", Systems Engineering and Electronics, vol. 26, no. 9, pp. 165-170, 2004.

Received: August 13, 2013

Revised: August 28, 2013

Accepted: August 28, 2013

(C) Zeng et al.; Licensee Bentham Open.

This is an open access article licensed under the terms of the Creative Commons Attribution Non-Commercial License (http://creativecommons.org/licenses/by-nc/3.0/) which permits unrestricted, non-commercial use, distribution and reproduction in any medium, provided the work is properly cited. 